Article

\title{
Wolkenstein's Model of Size Effects in CO Oxidation by Gold Nanoparticles
}

\author{
Nigora Turaeva * and Herman Krueger \\ Department of Biological Sciences, Webster University 470 E. Lockwood Ave., Webster Groves, MO 63119, USA; \\ hkrueger@webster.edu \\ * Correspondence: nigoraturaeva82@webster.edu; Tel.: +1-131-4246-7626
}

Received: 12 February 2020; Accepted: 21 February 2020; Published: 3 March 2020

\begin{abstract}
The Wolkenstein's theory of catalysis and the d-band theory of formation chemical bonds between transition metal catalysts and adsorbates were used to develop the approach applied to the kinetics of CO oxidation by gold nanoparticles. In the model, within the framework of the mechanism of the reaction going through dissociative adsorption of oxygen molecules and reaction with gas-phase $\mathrm{CO}$ molecules, weak and strong chemisorption states of intermediates $\left(\mathrm{O}, \mathrm{CO}_{2}\right)$ were taken into account in the kinetic equations by introducing reversible electronic steps corresponding to electron transfers between the intermediates and the catalyst. As a result, we obtain the expression for the reaction rate, which exhibits a volcano-shape dependence upon the size of the gold nanoparticles at the conditions when the intermediates fractions are not small compared to the empty active sites of the catalyst. It is supposed that the approach can be also applied to the Langmuir-Hinshelwood mechanism.
\end{abstract}

Keywords: CO oxidation; gold nanoparticles; fermi level; a volcano-shape plot; size effects

\section{Introduction}

Remarkable opportunities that nanotechnologies offer to the science and industry include a possibility of tuning properties of materials by their size and shape at the nanometer scale instead of replacement of materials by new ones to achieve desirable properties [1]. It is even more surprising when at the nanometer scale materials exhibit very interesting properties which are totally different from the behavior of the bulk counterparts. A very good example is gold, which is inert at the bulk but chemically active at the nanometer scale to catalyze many redox reactions at low temperatures [2-23]. One of such reactions catalyzed by gold nanocatalysts is the environmentally important CO oxidation reaction. It was experimentally shown that gold nanoparticles catalyze the reaction when their size is in the range of $1-5 \mathrm{~nm}$ [3-12], proceeding through the maximum rate at sizes around 2-3 nm. Other factors that contributed to the chemical activity of gold nanocatalysts with respect to $\mathrm{CO}$ oxidation are supports $[5,6,10,11]$, low coordinated surface atoms [8-10], surface defects $[14,15]$, quantum confinement of gold nanoparticles [5], and hot electrons $[17,18]$. Intensive theoretical and computational research of the size effects $[8-10,14-16,24,25]$ revealed many important aspects of the reaction, including the role of low coordination of surface atoms, adsorption energies, support effects, the detailed reaction paths, but the origin of the size dependence of the reaction rate has not been completely established. In the present study, we will investigate the possibility of obtaining a volcano-shape size dependence of the reaction rate by combining the Wolkenstein's theory of catalysis [26,27] based on a regulatory role of the Fermi level of catalysts and d-band model $[28,29]$ establishing the origin of the chemical bonds between intermediates and transition metal catalysts. 


\section{Wolkenstein's Approach}

Wolkenstein Th. [26,27] developed an electronic theory of catalytic reactions for semiconducting bulk catalysts. In the theory, chemisorbed particles introduce localized energy levels into the bandgap of the semiconductor in analogy with structural defects. Depending on energies of the localized states, they can trap electrons from the conducting band or holes from the valence band. The localization of electrons and holes on the chemisorbed intermediates transfer them from weak into strong chemisorbed states, as a result of which strong-acceptor and strong-donor states of intermediates are correspondingly established. Note that the electron transfers are regulated by the Fermi level, which is considered to be an energy level occupied by an electron with the probability of $1 / 2$. For pure semiconductors, it is in the middle of the bandgap. The occupancy of the localized energy states of intermediates adsorbed on the surface by electrons or holes obeys to the Fermi-Dirac statistics. Thus, the fractions of the intermediates in weak and strong chemisorbed states on the surface of the catalysts at the electronic equilibrium are determined by the Fermi level. Moreover, the electronic theory shows that the Fermi level can also regulate the selectivity of the catalyst with respect to concurrently proceeding reactions.

The Wolkenstein's theory developed for semiconductors can be directly applied to dielectric materials. However, for metallic catalysts, especially transition metals, it cannot be automatically used since there is no bandgap in metals, and the origin of chemical bonds between the intermediates and the metal catalysts is different. To overcome the limitation of the electronic theory to be applicable to transition metals, we will use the d-band model [28,29], explaining the origination of formation of chemical bonds between the molecular orbitals of adsorbates and the d-band energy states of transition metals. The fractions of reactive intermediates which are in strong chemisorbed states will be determined by energy positions of antibonding states formed between the LUMOs of molecules and the center of the d-band with respect to the Fermi level. To obtain the dependence of the reaction rate upon the Fermi level, we will solve kinetic equations for intermediates by taking into account electronic steps corresponding to electron transfers between weak and strong chemisorbed states within the framework of Eley-Rideal mechanism of CO oxidation. For metal clusters, we can use the ionization potential as the Fermi level which is the highest occupied energy level. To establish the size dependence of the reaction rate, we will use the jellium model $[30,31]$, which defines the ionization potential of spherical metal clusters as a function of the size.

\subsection{Jellium Model}

The jellium model of metal clusters [30,31] considers delocalized valence electrons to make the quantized motion in the uniform positively charged background by neglecting ionic structure. In the semiclassical approximation, which connect the properties of large clusters with the bulk surface properties, the asymptotic expressions for ionization potential and electron affinity of a spherical cluster with radius $r$ can be written based on the leptodermous expansion of total binding energy:

$$
\begin{aligned}
& I(R)=W_{b}+\alpha \frac{e^{2}}{4 \pi \varepsilon_{0} r}+o\left(r^{-2}\right) \\
& A(R)=W_{b}-\beta \frac{e^{2}}{4 \pi \varepsilon_{0} r}+o\left(r^{-2}\right)
\end{aligned}
$$

Here, $W_{\mathrm{b}}$ is the bulk work function, $\varepsilon_{0}$ is the permittivity of vacuum, $\alpha, \beta$ are the parameters, which are equal to $3 / 8$ and $5 / 8$, respectively. The higher-order terms (indicated by dots) are considered for very small clusters with the number of atoms in the cluster $<100$. For the gold, the ionization potential can vary between the work function of the bulk, $5.3 \mathrm{eV}$, and ionization potential of $9.2 \mathrm{eV}$ for a single atom [1,32], so its chemical properties can be tuned over a wide range by variation of the cluster size. 


\subsection{D-Band Model}

The d-band model $[28,29]$ explains the bonding of an adsorbate to a surface of transition metals. According to the model, an adsorbate valence level coupled with the s and d states of the transition-metal surface in a different way. Coupling to the broad s states leads to a shift and broadening of the adsorbate states, which is almost the same for all transition metals. It contributes to the bonding as "weak chemisorption". However, coupling to the narrow d-band (to the center of the d-band) gives rise to bonding and antibonding states just as in a two-state system. The different strength of chemisorption by transition metals is defined by the filling of the antibonding states which depends upon the relative position of antibonding states in energy with the Fermi level of the metal. Since the antibonding states are always above the $\mathrm{d}$ states, the energy of the $\mathrm{d}$ states relative to the Fermi level is considered as a good indicator of the bond strength. The closer the $d$ states are in energy to the Fermi level, the higher the antibonding states are and the stronger the bond. If the antibonding state is filled by electrons transferred from the Fermi level of the metal, then the adsorption of the molecules is weak or does not occur. If the Fermi level is below the antibonding state, which is then empty, the molecule forms a strong chemical bond with the surface. The more antibonding states are empty, the more molecules are adsorbed onto the surface, and the larger the fraction of the adsorbed molecule with strong bonds.

Applying the d-band model and the jellium model results to transition-metal nanocatalysts, we can suppose that adsorption and desorption of atoms and molecules can be tuned by the size of nanoparticles. The volcano-shape size dependence of the reaction rate for $\mathrm{CO}$ oxidation observed in experiments can be interpreted by the changing of adsorption and desorption of molecules at changing the size of gold nanoparticles, as the Fermi level of the particles (ionization potential) moves down at decreasing the size. For bulk gold, adsorption of oxygen molecules is weak. When the Fermi level moves down below the antibonding states of the adsorbates at decreasing the size, the adsorption of the molecules is enhanced. The number of adsorbed molecules increases, as far as the more antibonding states are becoming empty. The desorption of the products occurs until the Fermi level is higher than the antibonding states of the products. Note here that bulk gold adsorption of $\mathrm{CO}_{2}$ is mostly of a physical character [33]. Further decrease in size leads to the impairment of desorption. Thus, there should be some optimal size range, when both adsorption and desorption occur, and the reaction rate is significant. For gold nanoparticles, $\mathrm{CO}$ molecules are effectively oxidized in the size range of $1-5 \mathrm{~nm}$. This qualitative discussion can be related to the well-known Sabatier and Norskov et al [1,34]. principle about the volcano-shaped plot of the turnover frequency of catalytic chemical reactions as a function of the binding energy of the reactants onto the catalyst surface. The principle supposes a compromise between adsorption and desorption at optimal binding energies.

By means of kinetic equations for reaction intermediates, the aforementioned size effects can be quantified. Note that the size dependence of the reaction rate was theoretically analyzed based on different activity of distinct active sites of the metal catalysts, including terraces, edges, corners [13,35]. Considerations of distinct activities of such sites allow an explanation not only of monotonic dependence of turnover frequency as a function of cluster size but also a volcano-shape dependence [35]. Although such models give analytical expressions for the reaction rate as a function of cluster size, they are based on the assumption that the overall rate is a sum of terms contributing from fractions of different active sites, and the models do not take into account electronic transfers between the reagents (products) and the catalyst particle, responsible for the processes such as adsorption of reagents and desorption of products. In this study, based on weak and strong chemisorption concepts and the d-band theory, we obtain an analytic expression for the reaction rate as a function of cluster size in the framework of the mechanism of the reaction through dissociative adsorption of $\mathrm{O}_{2}$ molecules which becomes energetically favorable at the nanometer range [10] and interaction with gas-phase CO molecules (Eley-Rideal mechanism). We demonstrate a volcano-shape size dependence, when there is a competition between intermediates for the empty sites of the catalysts, and a monotonic size dependence in the case of abundancy of active sites. Note that $\mathrm{CO}$ oxidation for supported $\mathrm{Au}$ catalysts [19-23] and nanocomposite $\mathrm{MnCeO}_{x}$ catalyst [36-38] occurs favorably through adsorption 
and activation of both reagents $\left(\mathrm{O}_{2}\right.$ and $\left.\mathrm{CO}\right)$ (Langmuir-Hinshelwood mechanism). We suppose that the presented approach can be also applied to the Langmuir-Hinshelwood mechanism. In the Appendix A, it is shown that no volcano-shape dependence is observed for this mechanism in the case of abundancy of active sites on the catalyst. However, a volcano-shape dependence can be observed if fractions of intermediates of reagents and products on the surface are not small in comparison with empty active sites of the catalyst.

\subsection{Theoretical Model}

We will consider the mechanism of $\mathrm{CO}$ oxidation proceeding through dissociative adsorption of oxygen molecules onto the surface of the catalysts, interaction of atomic oxygen adsorbates with gas-phase $\mathrm{CO}$ molecules to form $\mathrm{CO}_{2}$ molecules and their desorption. Based on the Wolkenstein's approach discussed above, we include into this scheme two electronic reversible steps $\left(1^{*}\right.$ and $\left.2^{*}\right)$, which describe transitions between weak and strong chemisorption of oxygen and $\mathrm{CO}_{2}$ intermediates (Table 1).

Table 1. The Eley-Rideal mechanism of $\mathrm{CO}$ oxidation with two electronic steps. ( $\mathrm{Z}$ is an empty active site of the catalyst).

\begin{tabular}{|c|c|}
\hline Mechanism of CO Oxidation with Electro & \\
\hline $\mathrm{O}_{2}+2 \mathrm{Z} \rightleftarrows 2 \mathrm{ZO}^{0}$ & (1) \\
\hline $\mathrm{ZO}^{0} \rightleftarrows \mathrm{ZO}^{-}$ & $\left(1^{*}\right)$ \\
\hline 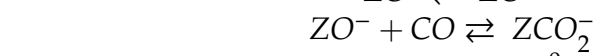 & (2) \\
\hline $\mathrm{ZCO}_{2}^{-} \rightleftarrows \mathrm{ZCO}_{2}^{0}$ & $\left(2^{*}\right)$ \\
\hline $\mathrm{ZCO}_{2}^{0} \rightarrow \mathrm{CO}_{2}+\mathrm{Z}$ & (3) \\
\hline
\end{tabular}

Oxygen intermediates are activated by accepting electrons from the catalyst. In the ion-radical state, they react with $\mathrm{CO}$ molecules from air, and ion-radicals of $\mathrm{CO}_{2}$ molecules are formed. As soon as $\mathrm{CO}_{2}$ intermediates are neutralized by donating electrons to the catalyst, they desorb from the surface of the catalyst.

We suppose that the electronic equilibrium is established much faster than that of molecules, and therefore the intermediate fractions in weak and strong chemisorption states are characterized by their equilibrium values. We can define the fractions of the intermediates as follows [25,26]:

$$
\eta_{\mathrm{O}}^{-}=\frac{N_{\mathrm{O}}^{-}}{N_{\mathrm{O}}^{-}+N_{\mathrm{O}}^{0}} ; \eta_{\mathrm{O}}^{0}=\frac{N_{\mathrm{O}}^{0}}{N_{\mathrm{O}}^{-}+N_{\mathrm{O}}^{0}} ; \eta_{\mathrm{CO} 2}^{-}=\frac{N_{\mathrm{CO} 2}^{-}}{N_{\mathrm{CO} 2}^{-}+N_{\mathrm{CO} 2}^{0}} ; \eta_{\mathrm{CO} 2}^{0}=\frac{N_{\mathrm{CO} 2}^{0}}{N_{\mathrm{CO} 2}^{-}+N_{\mathrm{CO} 2}^{0}}
$$

where $N^{0}$ and $N^{-}$are the concentrations of adsorbates in weak and strong chemisorption, correspondingly.

Based on the three-step mechanism with two electronic steps (Table 1), we can write the following kinetic equations for atomic oxygen and $\mathrm{CO}_{2}$ molecule intermediates:

$$
\begin{gathered}
\frac{d N_{O}}{d t}=k_{1} P_{\mathrm{O}_{2}}\left(1-s\left(N_{\mathrm{O}}+N_{\mathrm{CO} 2}\right)\right)^{2}-2 k_{-1}\left(N_{\mathrm{O}}^{0}\right)^{2}-k_{2} N_{\mathrm{O}}^{-} P_{\mathrm{CO}}+k_{-2} N_{\mathrm{CO} 2}^{-} \\
\frac{d N_{\mathrm{CO} 2}}{d t}=k_{2} N_{\mathrm{O}}^{-} P_{\mathrm{CO}}-k_{-2} N_{\mathrm{CO} 2}^{-}-k_{3} N_{\mathrm{CO} 2}^{0} .
\end{gathered}
$$

Here is the effective surface area of an active site of the catalyst, $\mathrm{P}$ is the reagent pressure. We can rewrite Equations (4) and (5) in the following way at the quasi-steady state condition:

$$
\begin{gathered}
k_{1} P_{\mathrm{O}_{2}}\left(1-s\left(N_{\mathrm{O}}+N_{\mathrm{CO} 2}\right)\right)^{2}-2 k_{-1}\left(N_{\mathrm{O}}^{0}\right)^{2}-k_{2} N_{\mathrm{O}}^{-} P_{\mathrm{CO}}+k_{-2} N_{\mathrm{CO} 2}^{-}=0 \\
k_{2} N_{\mathrm{O}}^{-} P_{\mathrm{CO}}-k_{-2} N_{\mathrm{CO} 2}^{-}-k_{3} N_{\mathrm{CO} 2}^{0}=0
\end{gathered}
$$


We can find $N_{0}$ by combining Equations (6) and (7) and taking into account Equation (3):

$$
N_{O}=\frac{1-s N_{C O 2}}{s+\eta_{O}^{0} \sqrt{\frac{2 k_{-1}}{k_{1} P_{O}}}}
$$

For simplicity of calculations, we assumed that desorption of products proceeds much slower than desorption of oxygen $\left(2 k_{-1}\left(N_{O}^{0}\right)^{2} \gg k_{3} N_{\mathrm{CO} 2}^{0}\right)$. Inserting Equation (8) into Equation (7), the following formula for the reaction rate can be obtained:

$$
R=k_{3} N_{\mathrm{CO} 2}^{0}=\frac{k_{2} k_{3} P_{\mathrm{CO}}}{k_{2} P_{\mathrm{CO}} \frac{1}{\eta_{\mathrm{CO} 2}^{0}}+\frac{1}{\eta_{\mathrm{O}}^{-}}\left(s+\eta_{\mathrm{O}}^{0} \sqrt{\frac{2 k_{-1}}{k_{1} P_{\mathrm{O} 2}}}\right)\left(k_{-2} \frac{\eta_{\mathrm{CO} 2}^{-}}{\eta_{\mathrm{CO} 2}^{0}}+k_{3}\right)}
$$

As we discussed above, the fractions of weak and strong intermediates can be determined by the energy of antibonding states formed between the LUMO states of the adsorbate and the d-band center of the catalyst with respect to the Fermi level. We can establish the relationships using the Fermi-Dirac statistics and the concepts of the d-band model as follows:

$$
\begin{gathered}
\eta_{O}^{-}=\frac{K_{1 *}}{K_{1 *}+1}=\frac{1}{1+e^{-\left(E_{f}-E_{O}^{a}\right) / k_{B} T}} \\
\eta_{O}^{0}=\frac{1}{1+K_{1 *}}=\frac{1}{1+e^{\left(E_{f}-E_{O}^{a}\right) / k_{B} T}} \\
\eta_{C O 2}^{-}=\frac{1}{1+K_{2 *}}=\frac{1}{1+e^{-\left(E_{f}-E_{\mathrm{CO} 2}^{a}\right) / k_{B} T}} \\
\eta_{\mathrm{CO} 2}^{0}=\frac{1}{K_{2 *}+1}=\frac{1}{1+e^{\left(E_{f}-E_{\mathrm{CO} 2}^{a}\right) / k_{B} T}} \\
\frac{\eta_{O}^{0}}{\eta_{O}^{-}}=\frac{1}{K_{1 *}}=e^{-\left(E_{f}-E_{O}^{a}\right) / k_{B} T} \\
\frac{\eta_{\mathrm{CO} 2}^{-}}{\eta_{\mathrm{CO} 2}^{0}}=\frac{1}{K_{2 *}}=e^{\left(E_{f}-E_{\mathrm{CO} 2}^{a}\right) / k_{B} T}
\end{gathered}
$$

Here, $K_{1 *}$ and $K_{2}$ are the equilibrium constants of electronic steps $1^{*}$ and $2^{*}$, correspondingly, $E_{O}^{a}$ and $E_{\mathrm{CO} 2}^{a}$ are the energy levels of antibonding states of atomic oxygen and $\mathrm{CO}_{2}$ on the surface of the catalyst, respectively, $T$ is the temperature, $k_{\mathrm{B}}$ is the Boltzmann's constant. In order to take into account the support effect on the reaction rate, we can write the following expression for the effective Fermi level defined by Equation (1) by adding an additional term for the support, $E_{\text {sup }}$ :

$$
E_{f}=W_{b}+\alpha \frac{e^{2}}{4 \pi \varepsilon_{0} r}-E_{\text {sup }}
$$

Equation (16) implies that reducible supports, which lower the Fermi level, contribute to the adsorption of molecules while oxidizing supports, which raise the Fermi level, improve the desorption of the products. This agrees well with the experimental results on reducible supports $[11,14,16]$. Using Equations (10)-(15) and (16), we can rewrite Equation (9) as a function of the size of the particles:

$$
R=\frac{k_{2} k_{3} P_{C O}}{k_{2} P_{C O}\left(1+e^{\frac{\left(W_{b}+\alpha \frac{e^{2}}{4 \pi \varepsilon_{0} r}-E_{\text {sup }}-E_{C O 2}^{a}\right)}{k_{B} T}}\right)+\left(s\left(1+e^{-\frac{\left(W_{b}+\alpha \frac{e^{2}}{4 \pi \varepsilon_{0} r}-E_{\text {sup }}-E_{O}^{a}\right)}{k_{B} T}}\right)+e^{-\left(W_{b}+\alpha \frac{e^{2}}{4 \pi \varepsilon_{0} r}-E_{\text {sup }}-E_{O}^{a}\right) / k_{B} T} \sqrt{\frac{2 k_{-1}}{k_{1} P 2}}\right)\left(k_{-2} e^{\frac{W_{b}+\alpha \frac{e^{2}}{4 \pi \varepsilon_{0} r}-E_{\text {sup }}-E_{C O 2}^{a}}{k_{B} T}}+k_{3}\right)}
$$


From Equation (9), we can obtain the expression for the optimal Fermi level of the catalyst, at which the reaction rate is maximal by taking into account Equations (10)-(15):

$$
E_{f}^{o p t}=\frac{1}{2}\left(E_{\mathrm{CO} 2}^{a}+E_{O}^{a}\right)+\frac{k_{B} T}{2} \ln \left(\frac{k_{3}}{k_{2} P_{\mathrm{CO}}} \frac{1+\sqrt{2 k_{-1} / k_{1} P_{\mathrm{O} 2} S^{2}}}{1+k_{-2} / k_{2} P_{\mathrm{CO}}}\right)
$$

The optimal size can be then calculated using Equation (16):

$$
r^{o p t}=\frac{\alpha e^{2}}{4 \pi \varepsilon_{0}}\left[\frac{1}{E_{f}^{o p t}-W_{b}+E_{s}}\right]
$$

\section{Discussion}

In the framework of the presented approach, we can show that the size dependence of the reaction rate has a volcano-shape, which was experimentally established in a number of works [3-7]. Note here that an evaluation of numerical values of the reaction rate, the optimal Fermi level, and the optimal size is a difficult task. As it is seen from Equations (18) and (19), several parameters should be available, including energies of antibonding states of reagents and products with d-band center of the catalyst, reaction rate constants, pressure of reagents, temperature, and support contributions to the Fermi level of the catalyst. Therefore, we will qualitatively analyze the equations.

From Equation (17), it is seen that the reaction rate contains the exponential functions having both "+" and "-"signs in the exponents, meaning direct and inverse dependences on the size of the particle, correspondingly. The analysis of Equation (17) indicates that at decreasing size the Fermi level is lowered (its absolute value increases), and the reaction rate increases because it passes the energy level of the antibonding states of oxygen atoms. As a result, adsorption of oxygen molecules becomes stronger, and the fractions of oxygen intermediates increase, while desorption of $\mathrm{CO}_{2}$ molecules occurs fast (region a, Figure 1). In this region, the reaction rate is limited by adsorption of oxygen. Upon further decreasing the size, the Fermi level is lowered below the antibonding states of $\mathrm{CO}_{2}$ molecules, and desorption is impaired, while adsorption occurs fast, and the reaction rate is limited by desorption of the products (region b, Figure 1). Therefore, at the optimal size of the nanoparticles $\left(r^{\text {opt }}\right)$, the reaction rate takes its maximal value. To catalyze efficiently the reaction of $\mathrm{CO}$ oxidation, the size of the nanoparticle should be in the range between $r_{\mathrm{CO} 2}$ and $r_{\mathrm{O}}$, which corresponds to the Fermi levels of the catalyst, matching with energies of antibonding states of $\mathrm{CO}_{2}$ and oxygen atoms on the surface of the catalyst:

$$
\begin{aligned}
r_{\mathrm{CO} 2} & =\frac{\alpha e^{2}}{4 \pi \varepsilon_{0}\left(E_{\mathrm{CO} 2}^{a}+E_{\text {sup }}-W_{b}\right)} ; \\
r_{O} & =\frac{\alpha e^{2}}{4 \pi \varepsilon_{0}\left(E_{O}^{a}+E_{\text {sup }}-W_{b}\right)}
\end{aligned}
$$

In general, in Equation (17), the volcano-shape dependence is not symmetrical due to different coefficients of exponential functions with " - " and " + " signs. It is important to note that at the quasi steady-state, a volcano-shape dependence is observed if the intermediates are not small compared to empty active sites of the catalysts. However, in the case of abundancy of active sites $\left(\mathrm{s}\left(\mathrm{N}_{\mathrm{O}}+\mathrm{N}_{\mathrm{CO} 2}\right)<<1\right)$, Equation (17) is reduced, and the dependence of the reaction rate upon the size is monotonic:

$$
R=\frac{k_{2} k_{3} P_{C O}}{\sqrt{\frac{2 k_{-1}}{k_{1} P_{O 2}}} k_{-2} e^{\left(E_{O}^{a}-E_{C O 2}^{a}\right) / k_{B} T}+k_{3} e^{-\left(W_{b}+\alpha \frac{e^{2}}{4 \pi \varepsilon_{0} r^{r}}-E_{s u p}-E_{O}^{a}\right) / k_{B} T} .}
$$




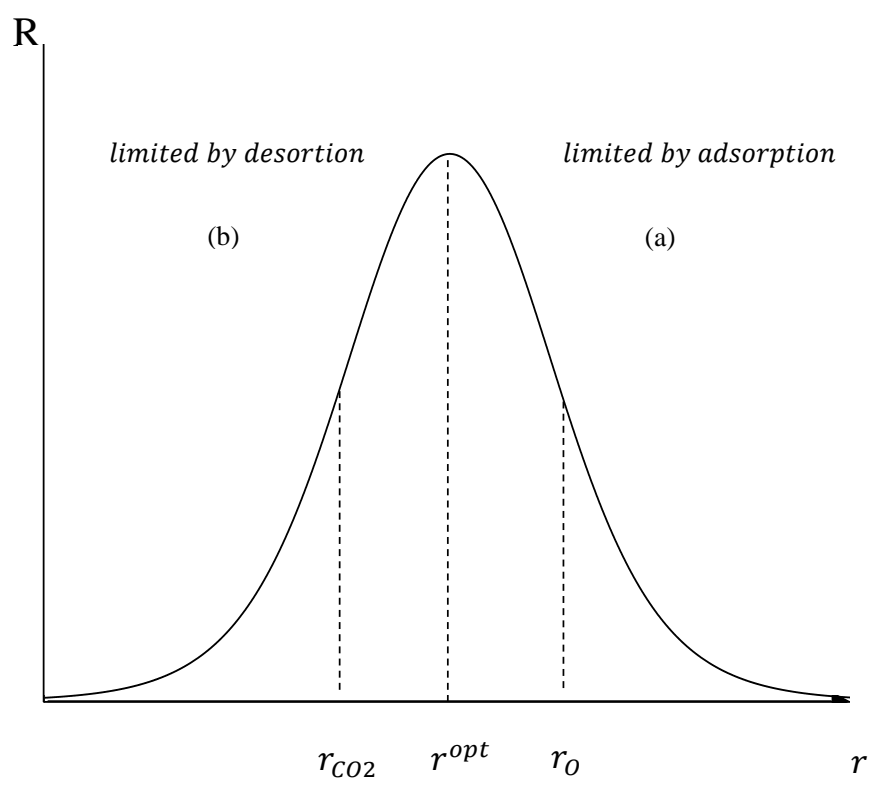

Figure 1. A volcano-shaped size dependence of $\mathrm{CO}$ oxidation rate for gold nanoparticles with adsorption (a) and desorption (b) rate limited steps.

Thus, we demonstrated a possibility of the volcano-shape size dependence of the reaction rate within the framework of the Eley-Rideal mechanism. However, the presented approach can be also applied to the Langmuir-Hinshelwood mechanism of the reaction proceeding through adsorption and activation of both reagents $\left(\mathrm{O}_{2}\right.$ and $\left.\mathrm{CO}\right)$. We can show that in the case of abundancy of active sites $(Z>>1)$, no volcano-shape size dependence is observed (see Appendix A). The volcano-shape size dependence becomes possible for this mechanism, if there is a competition of intermediates for the empty active sites on the surface of the catalyst. The latter case involves a quartic polynomial function, of which the analytical solution is complicated.

\section{Conclusions}

In summary, we applied the Wolkenstein's theory of catalytic reactions to $\mathrm{CO}$ oxidation reaction catalyzed by gold nanoparticles to demonstrate a possibility of a volcano-shaped size dependence of the reaction rate. The model is based on an important role of the Fermi level of the catalysts in determining the fractions of intermediates in weak and strong chemisorption states, which take part in different steps of the reaction path. To overcome the limit of application of the Wolkenstein's theory to metallic catalysts, we use qualitative concepts of the d-band model, explaining the origin of strong chemical bonds between the adsorbates and the surface of transition-metal catalysts. To determine the size dependence of the Fermi level, the size dependence of ionization potential is used which is obtained in semiclassical approximation of the jellium model by taking into account the support effect. According to the presented approach, the initial decrease of the size of the particle enhances the adsorption of the oxygen molecules, as a result of which the reaction rate increases. Further decreasing the size impairs the desorption of $\mathrm{CO}_{2}$ molecules leading to the decrease of the reaction rate. Thus, the gold nanoparticles catalyze the $\mathrm{CO}$ oxidation reaction with the maximum rate at some optimal size. The reducible and oxidizing supports contribute positively to the reaction rate by lowering or rising the Fermi level of the catalyst, leading to the enhancement of adsorption of reagents or desorption of products, respectively. The model presented in the work is developed for spherical clusters, for which Equation (1) is valid, but it is assumed it can be also applied to other shaped particles by introducing different coefficients in the expression for the ionization potential. 
Our analysis shows that for the observation of a volcano-shape size dependence, it is important that the intermediates of reagents and products should not be small in comparison with empty active sites on the surface of the catalyst.

Author Contributions: Conceptualization, N.T.; formal analysis, N.T. and H.K.; writing-original draft preparation, N.T.; writing-review and editing, H.K. and N.T. All authors have read and agree to the published version of the manuscript.

Funding: This research received no external funding.

Conflicts of Interest: The authors declare no conflict of interest.

\section{Appendix A}

\section{Langmuir-Hinshelwood mechanism.}

The mechanism of $\mathrm{CO}$ oxidation through adsorption of both reagents and interaction between them can be written by taking into account electron transfers between the intermediates and the catalyst in the following way:

$$
\begin{gathered}
\mathrm{O}_{2}+2 \mathrm{Z} \rightleftarrows 2 \mathrm{ZO}^{0} \\
\mathrm{ZO}^{0} \rightleftarrows \mathrm{ZO}^{-} \\
\mathrm{CO}+\mathrm{Z} \rightleftarrows \mathrm{ZCO}^{0} \\
\mathrm{ZCO}^{0} \rightleftarrows \mathrm{ZCO} \\
\mathrm{ZO}^{-}+\mathrm{ZCO}^{-} \rightleftarrows \mathrm{ZCO}_{2}^{-}+\mathrm{Z} \\
\mathrm{ZCO}_{2}^{-} \rightleftarrows \mathrm{ZCO}_{2}^{0} \\
\mathrm{ZCO}_{2}^{0} \longrightarrow \mathrm{CO}_{2}+\mathrm{Z}
\end{gathered}
$$

Here $\left(1^{*}\right),\left(2^{*}\right)$, and $\left(3^{*}\right)$ are electronic steps of the reaction. For the case of abundancy of active sites $(Z>>1)$, the following kinetic equations can be written by taking into account weak and strong chemisorption of both reagents and assuming that all electronic steps are in equilibrium:

$$
\begin{gathered}
\frac{d N_{O}}{d t}=k_{1} P_{\mathrm{O}_{2}}-k_{-1}\left(N_{\mathrm{O}}^{0}\right)^{2}-k_{3} N_{\mathrm{CO}}^{-} N_{\mathrm{O}}^{-}+k_{-3} N_{\mathrm{CO} 2}^{-} \\
\frac{d N_{\mathrm{CO}}}{d t}=k_{2} P_{\mathrm{CO}}-k_{-2} N_{\mathrm{CO}}^{0}-k_{3} N_{\mathrm{CO}}^{-} N_{\mathrm{O}}^{-}+k_{-3} N_{\mathrm{CO} 2}^{-} \\
\frac{d N_{\mathrm{CO} 2}}{d t}=k_{3} N_{\mathrm{CO}}^{-} N_{\mathrm{O}}^{-}-k_{-3} N_{\mathrm{CO} 2}^{-}-k_{4} N_{\mathrm{CO} 2}^{0}
\end{gathered}
$$

At the quasi steady-state conditions, they can be rewritten as:

$$
\begin{gathered}
k_{1} P_{\mathrm{O}_{2}}-k_{-1}\left(N_{\mathrm{O}}^{0}\right)^{2}-k_{3} N_{\mathrm{CO}}^{-} N_{O}^{-}+k_{-3} N_{\mathrm{CO} 2}^{-}=0 \\
k_{2} P_{\mathrm{CO}}-k_{-2} N_{\mathrm{CO}}^{0}-k_{3} N_{\mathrm{CO}}^{-} N_{\mathrm{O}}^{-}+k_{-3} N_{\mathrm{CO} 2}^{-}=0 \\
k_{3} N_{\mathrm{CO}}^{-} N_{\mathrm{O}}^{-}-k_{-3} N_{\mathrm{CO} 2}^{-}-k_{4} N_{\mathrm{CO} 2}^{0}=0
\end{gathered}
$$

We can introduce the definitions of the fractions of the intermediates in different chemisorbed states:

$$
\eta_{\mathrm{O}}^{-}=\frac{N_{\mathrm{O}}^{-}}{N_{\mathrm{O}}^{-}+N_{\mathrm{O}}^{0}} ; \eta_{\mathrm{O}}^{0}=\frac{N_{\mathrm{O}}^{0}}{N_{\mathrm{O}}^{-}+N_{\mathrm{O}}^{0}} \eta_{\mathrm{CO}}^{-}=\frac{N_{\mathrm{CO}}^{-}}{N_{\mathrm{CO}}^{-}+N_{\mathrm{CO}}^{0}} ; \eta_{\mathrm{CO}}^{0}=\frac{N_{\mathrm{CO}}^{0}}{N_{\mathrm{CO}}^{-}+N_{\mathrm{CO}}^{0}} ; \eta_{\mathrm{CO} 2}^{-}=\frac{N_{\mathrm{CO} 2}^{-}}{N_{\mathrm{CO} 2}^{-}+N_{\mathrm{CO} 2}^{0}} ; \eta_{\mathrm{CO} 2}^{0}=\frac{N_{\mathrm{CO} 2}^{0}}{N_{\mathrm{CO} 2}^{-}+N_{\mathrm{CO} 2}^{0}}
$$

By combining Equations (A4) and (A6) and assuming $k_{4} N_{\mathrm{CO} 2}^{0} \ll k_{-1}\left(N_{\mathrm{O}}^{0}\right)^{2}$, we can find $N_{\mathrm{O}}^{-}$using the expressions (A7):

$$
N_{O}^{-}=\frac{\eta_{O}^{-}}{\eta_{O}^{0}} \sqrt{\frac{k_{1}}{k_{-1}} P_{O 2}}
$$


By solving Equation (A5) for $N_{C O}^{-}$we have the following expression:

$$
N_{\mathrm{CO}}^{-}=\frac{\eta_{\mathrm{CO}}^{-}}{\eta_{\mathrm{CO}}^{0}}\left(\frac{k_{2}}{k_{-2}} P_{\mathrm{CO}}-\frac{1}{k_{-2}} R\right)
$$

Inserting Equations (A8) and (A9) into Equation (A6), the expression for the rate is obtained:

$$
R=\frac{\sqrt{k_{1}} k_{2} k_{3} k_{4} P_{\mathrm{CO}} \sqrt{P_{\mathrm{O} 2}}}{\sqrt{k_{1}} k_{3} k_{4} \sqrt{P_{\mathrm{O} 2}}+\sqrt{k_{-1}} k_{-2} k_{4} \frac{\eta_{\mathrm{CO}}^{0}}{\eta_{\mathrm{CO}}^{-}} \frac{\eta_{O}^{0}}{\eta_{O}^{-}}+\sqrt{k_{-1}} k_{-2} k_{-3} \frac{\eta_{\mathrm{CO}}^{0}}{\eta_{\mathrm{CO}}^{-}} \frac{\eta_{\mathrm{O}}^{0}}{\eta_{\mathrm{O}}^{-}} \frac{\eta_{\mathrm{CO}}^{-}}{\eta_{\mathrm{CO} 2}^{0}}}
$$

The fractions of the intermediates are defined as a function of energy difference between the antibonding states of the intermediates and the Fermi level of the gold nanoparticle:

$$
\frac{\eta_{O}^{0}}{\eta_{O}^{-}}=e^{-\left(E_{f}-E_{O}^{a}\right) / k T} ; \frac{\eta_{\mathrm{CO}}^{0}}{\eta_{\mathrm{CO}}^{-}}=e^{-\left(E_{f}-E_{C O}^{a}\right) / k T} ; \frac{\eta_{\mathrm{CO} 2}^{-}}{\eta_{\mathrm{CO} 2}^{0}}=e^{\left(E_{f}-E_{C O 2}^{a}\right) / k T}
$$

Here, $E_{\mathrm{O}^{\prime}}^{a} E_{\mathrm{CO}}^{a}$, and $E_{\mathrm{CO} 2}^{a}$ are the energies of antibonding states of $\mathrm{O}, \mathrm{CO}$, and $\mathrm{CO}_{2}$ intermediates, respectively. Combination of Equations (A10) and (A11) gives the following dependence of the reaction rate on the Fermi level:

$$
R=\frac{\sqrt{k_{1}} k_{2} k_{3} k_{4} P_{\mathrm{CO}} \sqrt{P_{\mathrm{O} 2}}}{\sqrt{k_{1}} k_{3} k_{4} \sqrt{P_{\mathrm{O} 2}}+\sqrt{k_{-1}} k_{-2} k_{4} e^{-\frac{\left(2 E_{f}-E_{O}^{a}-E_{C O}^{a}\right)}{k T}}+\sqrt{k_{-1}} k_{-2} k_{-3} e^{-\frac{\left(E_{f}-E_{O}^{a}-E_{C O}^{a}+E_{C O 2}^{a}\right)}{k T}}}
$$

From Equation (A12), it is seen that the dependence of the rate upon the Fermi level of the catalyst is monotonic, and it increases with lowering the Fermi level. Thus, in the case of abundancy of active sites, upon decreasing the size of the particles the rate increases due to enhanced adsorption of reagents.

\section{References}

1. Roduner, E. Size matters: Why nanomaterials are different. Chem. Soc. Rev. 2006, 35, 583. [CrossRef] [PubMed]

2. Hutchings, G.J.; Haruta, M.A. Golden age of catalysis: A perspective. Appl. Catal. A 2005, 291, 2. [CrossRef]

3. Haruta, M. Catalysis: Gold rush. Nature 2005, 437, 1098. [CrossRef] [PubMed]

4. Haruta, M.; Kobayashi, T.; Sano, H.; Yamada, N. Novel gold catalysts for the oxidation of carbon monoxide at a temperature far below $0{ }^{\circ} \mathrm{C}$. Chem. Lett. 1987, 16, 405-408. [CrossRef]

5. Valden, M.; Lai, X.; Goodman, D.W. Onset of catalytic activity of gold clusters on titania with the appearance of nonmetallic properties. Science 1998, 281, 1647. [CrossRef] [PubMed]

6. Valden, M.; Pak, S.; Lai, X.; Goodman, D.W. Structure sensitivity of $\mathrm{CO}$ oxidation over model $\mathrm{Au} / \mathrm{TiO}_{2}$ catalysts. Catal. Lett. 1998, 56, 7. [CrossRef]

7. Haruta, M. Gold as a low-temperature oxidation catalyst: Factors controlling activity and selectivity. Stud. Surf. Sci. Catal. 1997, 110, 123.

8. Lopez, N.; Janssens, T.V.W.; Clausen, B.S.; Xu, Y.; Mavrikakis, M.; Bligaard, T.; Nørskov, J.K. On the origin of the catalytic activity of gold nanoparticles for low-temperature CO oxidation. J. Catal. 2004, 223, 232-235. [CrossRef]

9. Hammer, B.; Nørskov, J.K. Why gold is the noblest of all metals. Nature 1995, 376, 2238. [CrossRef]

10. Kleis, J.; Greeley, J.; Romero, N.A.; Morozov, V.A.; Falsig, H.; Larsen, A.H.; Lu, J.; Mortensen, J.J.; Dułak, M.; Nørskov, J.K.; et al. Finite size effects in chemical bonding: From small clusters to solids. Catal. Lett. 2011, 141, 1067-1071. [CrossRef]

11. Janssens, T.V.; Clausen, B.S.; Hvolbæk, B.; Falsig, H.; Christensen, C.H.; Bligaard, T.; Nørskov, J.K. Insights into the reactivity of supported Au nanoparticles: Combining theory and experiments. Top. Catal. 2007, 44, 15. [CrossRef] 
12. Schimpf, S.; Lucas, M.; Mohr, C.; Rodemerck, U.; Brückner, A.; Radnik, J.; Hofmeister, H.; Claus, P. Supported gold nanoparticles: In-depth catalyst characterization and application in hydrogenation and oxidation reactions. Catal. Today 2002, 72, 63-78. [CrossRef]

13. Rashkeev, S.N.; Lupini, A.R.; Overbury, S.H.; Pennycook, S.J.; Pantelides, S.T. Role of the nanoscale in catalytic CO oxidation by supported Au and Pt nanostructures. Phys. Rev. B 2007, 76, 035438. [CrossRef]

14. Landman, U.; Yoon, B.; Zhang, C.; Heiz, U.; Arenz, M. Factors in gold nanocatalysis: Oxidation of CO in the non-scalable size regime. Top. Catal. 2007, 44, 145-158. [CrossRef]

15. Arenz, M.; Landman, U.; Heiz, U. CO Combustion on Supported Gold Clusters. ChemPhysChem 2006, 7 , 1871-1879. [CrossRef] [PubMed]

16. Chen, M.; Goodman, D.W. Catalytically active gold: From nanoparticles to ultrathin films. Acc. Chem. Res. 2006, 39, 739-746. [CrossRef] [PubMed]

17. Kim, S.M.; Lee, H.; Goddeti, K.C.; Kim, S.H.; Park, J.Y. Photon-induced hot electron effect on the catalytic activity of ceria-supported gold nanoparticles. J. Phys. Chem. C 2015, 119, 16020-16025. [CrossRef]

18. Park, J.Y.; Baker, L.R.; Somorjai, G.A. Role of hot electrons and metal-oxide interfaces in surface chemistry and catalytic reactions. Chem. Rev. 2015, 115, 2781-2817. [CrossRef]

19. Yang, B.; Peng, A.; Wang, X.; Huang, Q.; Chen, M.; Shen, Y.; Xu, H.; Zhu, S. Simultaneous catalytic oxidation of $\mathrm{CO}$ and $\mathrm{Hg}^{0}$ over $\mathrm{Au} / \mathrm{TiO}_{2}$ catalysts: Structure and mechanism study. Mol. Catal. 2019, 479, 110633. [CrossRef]

20. Saqlain, M.A.; Hussain, A.; Siddiq, M.; Leitão, A.A. A DFT+U study of the Mars Van Krevelen mechanism of $\mathrm{CO}$ oxidation on $\mathrm{Au} / \mathrm{TiO}_{2}$ catalysts. Appl. Catal. A Gen. 2016, 519, 27-33. [CrossRef]

21. Qian, K.; Huang, W.; Fang, J.; Lv, S.; He, B.; Jiang, Z.; Wei, S. Low-temperature CO oxidation over Au/ZnO/SiO 2 catalysts: Some mechanism insights. J. Catal. 2008, 255, 269-278. [CrossRef]

22. Duan, D.; Hao, C.; Shi, W.; Wang, H.; Ma, C.; Song, X.; Sun, Z. Au/CeO 2 nanorods modified by $\mathrm{TiO}_{2}$ through a combining dealloying and calcining method for low-temperature CO oxidation. Appl. Surf. Sci. 2019, 484, 354-364. [CrossRef]

23. Arena, F.; Famulari, P.; Interdonato, N.; Bonura, G.; Frusteri, F.; Spadaro, L. Physico-chemical properties and reactivity of $\mathrm{Au} / \mathrm{CeO} 2$ catalysts in total and selectove oxidation of CO. Catal. Today 2006, 116, 384-390. [CrossRef]

24. Turaeva, N.; Preuss, M. Electronic effects in CO oxidation by nanoparticles catalysts. Catal. Commun. 2015, 65, 30-33. [CrossRef]

25. Turaeva, N.; Krueger, H. The vibronic theory of size effects in CO oxidation by gold nanocatalysts. Catal. Lett. 2017, 147, 2100-2105. [CrossRef]

26. Volkenstein, F.F. The Electron Theory of Catalysis on Semiconductors; McMillan: New York, NY, USA, 1963.

27. Wolkenstein, T. The electron theory of catalysis on semiconductors. In Advances in Catalysis; Academic Press: New York, NY, USA, 1960; Volume 12, pp. 189-264.

28. Hammer, B.; Nørskov, J.K. Theoretical Surface Science and Catalysis-Calculations and Concepts. Adv. Catal. 2000, 45, 71-129.

29. Norskov, J.K.; Abild-Pedersen, F.; Studt, F.; Bligaard, T. Density functional theory in surface chemistry and catalysis. Proc. Natl. Acad. Sci. USA 2011, 108, 937-943. [CrossRef] [PubMed]

30. Brack, M. The physics of simple metal clusters: Self-consistent jellium model and semiclassical approaches. Rev. Mod. Phys. 1993, 65, 677. [CrossRef]

31. Perdew, J. Energetics of charged metallic particles: From atom to bulk solid. Phys. Rev. B 1988, $37,6175$. [CrossRef]

32. Scanlon, M.D.; Peljo, P.; Méndez, M.A.; Smirnov, E.; Girault, H.H. Charging and discharging at the nanoscale: Fermi level equilibration of metallic nanoparticles. Chem. Sci. 2015, 6, 2705-2720. [CrossRef]

33. Zhang, G.; Musgrave, C.B. Comparison of DFT methods for molecular orbital eigenvalue calculations. J. Phys. Chem. A 2007, 111, 1554-1561. [CrossRef] [PubMed]

34. Jacobsen, C.J.; Dahl, S.; Clausen, B.S.; Bahn, S.; Logadottir, A.; Nørskov, J.K. Catalyst design by interpolation in the periodic table: Bimetallic ammonia synthesis catalysts. J. Am. Chem. Soc. 2001, 123, 8404-8405. [CrossRef]

35. Murzin, D.Y. Cluster size dependent kinetics: Analysis of different reaction mechanisms. Catal. Lett. 2015, 145, 1948-1954. [CrossRef] 
36. Arena, F.; di Chio, R.; Espro, C.; Palella, A.; Spadaro, L. A definitive assessment of the CO oxidation pattern of a nanocomposite $\mathrm{MnCeO}_{\mathrm{x}}$ catalyst. React. Chem. Eng. 2018, 3, 293-300. [CrossRef]

37. Arena, F.; Di Chio, R.; Filiciotto, L.; Trunfio, G.; Espro, C.; Palella, A.; Patti, A.; Spadaro, L. Probing the functionality of nanostructured $\mathrm{MnCeOx}$ catalysts in the carbon monoxide oxidation: Part II. Reaction mechanism and kinetic modelling. Appl. Catal. B 2017, 218, 803-809. [CrossRef]

38. Arena, F.; Di Chio, R.; Fazio, B.; Espro, C.; Spiccia, L.; Palella, A.; Spadaro, L. Probing the functionality of nanostructured $\mathrm{MnCeOx}$ catalysts in the carbon monoxide oxidation: Part 1. Influence of cerium addition on structure and CO oxidation activity. Appl. Catal. B 2017, 210, 14-22. [CrossRef]

(C) 2020 by the authors. Licensee MDPI, Basel, Switzerland. This article is an open access article distributed under the terms and conditions of the Creative Commons Attribution (CC BY) license (http://creativecommons.org/licenses/by/4.0/). 